\title{
Diseño de un sistema electrónico para el bloqueo de vehículos de transporte público basados en la medida de \\ alcohol por litro de sangre en los conductores
}

Design of an electronic system for blocking public transport vehicles based on the measurement of alcohol per liter of blood in drivers.

Cristhian Andres Villafuerte Haro. ${ }^{1}$, Silvia Catalina Villacís Torres. ${ }^{2}$ \& Víctor Eduardo Villafuerte Haro. ${ }^{3}$

\begin{abstract}
.
DOI: https://doi.org/10.33262/cienciadigital.v3i3.3.801

The aim of this research work was to design an electronic system for the blocking of public transport vehicles based on the measurement of alcohol per liter of blood in drivers. For the development of the system, an MQ-3 alcohol sensor was included that allowed measuring the level of alcohol and an Arduino that is the brain of the device, coupled in a structure, which was developed in SolidWorks and developed in a 3D printer. The methodology used is of analytical approach since we start from a whole that in this case are traffic accidents, in this way the causes that originated and deductive were known because the hypothesis was linked to the results collected from the execution of the device and synthetic since at the end of the test bench and feasibility assessments conclusions and recommendations could be issued. As a result, it was obtained that the measurement accuracy of the electronic system is reliable for the measurement of alcohol in people and can be implemented through national public
\end{abstract}

\footnotetext{
${ }^{1}$ Escuela Superior Politécnica de Chimborazo, maestría en Transporte y Logística. Riobamba, Ecuador. cristhian16_17@hotmail.com

2 Instituto Superior Tecnológico Cotopaxi, Tecnología Superior en Logística Multimodal. Latacunga, Ecuador. cattyvt1977@ hotmail.com

${ }^{3}$ Escuela Superior Politécnica de Chimborazo, Facultad Informática y Electrónica. Riobamba, Ecuador. victoredu1998@hotmail.com
} 
policies that supported the development of the project. It is recommended to monitor urban public transport drivers in terms of consumption of alcoholic beverages during their work days, to protect the safety of users and other road actors.

Keywords: Engineering and transport technology, Electronic system, Monitoring of sing, Drivers, Public transportation, Alcoholmeter, Alcohol sensor, Blocking of vehicles.

\section{Resumen.}

El objetivo del presente trabajo de investigación fue la de diseñar un sistema electrónico para el bloqueo de vehículos de transporte público basados en la medida de alcohol por litro de sangre en los conductores. Para el desarrollo del sistema se incluyó un sensor de alcohol MQ-3 mismo que permitió medir el nivel de alcohol y un Arduino que es el cerebro del dispositivo, acoplada en una estructura, la cual fue desarrollada en SolidWorks y elaborada en una impresora 3D. La metodología empleada es de enfoque analítico puesto que partimos de un todo que en este caso son los accidentes de tránsito, de esta manera se conoció las causas que lo originan y deductivo pues se vinculó la hipótesis planteada con los resultados recogidos de la ejecución del dispositivo y sintético ya que al finalizar con el banco de pruebas y evaluaciones de factibilidad se pudo emitir conclusiones y recomendaciones. Como resultados se obtuvo que la precisión de la medición del sistema electrónico es fiable para la medición del alcohol en las personas y puede ser implementado mediante políticas públicas nacionales que respaldaron la elaboración del proyecto. Se recomienda monitorear a los conductores de transporte público urbano en materia de consumo de bebidas alcohólicas durante sus jornadas de trabajo, para precautelar la segura de los usuarios y demás actores viales.

Palabras claves: Ingeniería y tecnología del transporte, Sistema electrónico, Monitoreo de signos, Conductores, Transporte público, Alcoholímetro, Sensor de alcohol, Bloqueo de vehículos,

\section{Introducción.}

La invención tecnológica tanto en dispositivos electrónicos como la Internet, ha dado paso a la transmisión de información, facilitando el correcto cumplimiento de actividades cotidianas y específicas, la relevancia de estar interconectados para el procesamiento de datos, usar sensores que recolecten información para procesarlas y de manera conjunta presentarlas para fines más técnicos y específicos utilizando medios de comunicación inalámbrica en lo cual se basa este proyecto de investigación. El desarrollo de la tecnología en un eje tan estratégico para el país como es la movilidad y en la misma se tiene graves problemas como el 
irresponsable irrespeto a las leyes de tránsito e irresponsabilidad en la conducta de los conductores han provocado una gran cantidad de siniestros viales que dan como resulta la muerte de sí mismo como la de muchas personas.

\section{Metodologia.}

Se define que la presente investigación es cuasi experimental debido a que el $50 \%$ de la investigación es en laboratorio y el otro $50 \%$ realizar un banco de pruebas aplicando el dispositivo electrónico a la realidad en vehículos de transporte de transporte público. La investigación es de tipo transversal pues se recogerán datos en un punto específico del tiempo de la población. Para lo cual, se consideró que la unidad de análisis corresponde a la entidad representativa que va a ser objeto específico de estudio en una medición, y se refiere al que es objeto de interés en la realización de esta investigación. En el caso del proyecto, la unidad de análisis son los conductores de transporte de buses urbanos legalmente constituidos en la Ciudad de Riobamba sobre el cual se van hacer los controles.

De la población de estudio señalada, se definió que el tamaño de la muestra es de 109 personas, misma que resultó de la aplicación de la fórmula planteada por (Goode \& Hatt, 2004), a quienes se aplicó las técnicas e instrumentos de recolección de datos, en este caso la encuesta, misma que fue validada previo su aplicación total a través del Alpha de Cronbac.

\section{Resultados.}

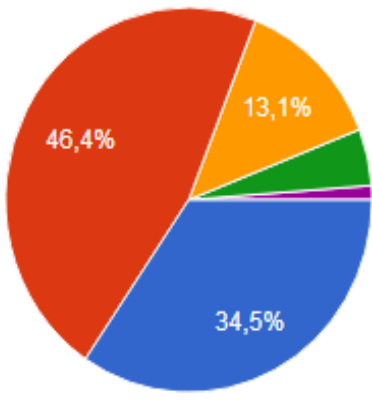

Nunca (0)

Una o menos veces al mes (1)

2 a 4 veces al mes (2)

203 veces a la semana (3)

4 o más veces a la semana (4)

Figura 1 Frecuencia de consumo

Fuente: Cristhian Villafuerte, 2018.

El $46 \%$ de los conductores encuestados afirman que consumen al menos una vez al mes alcohol el $31,5 \%$ no consume alcohol, pero lo más importante es que un $13,5 \%$ los conductores beben de 2 a 4 veces al mes que se considera muy habitual el consumo de bebidas alcohólicas y un número considerable de conductores con una frecuencia de 2 a 4 veces a la semana.

Los conectores que consumen bebidas alcohólicas el 49,4\% de los encuestados solo beben entre tres o 4 vasos de licor y un $47 \%$ apenas uno o dos vasos cada vez que toma sin embargo 
el tercer lugar hay conductores que toman de cinco a seis vasos lo cual se considera grandes cantidades de ingesta de alcohol.

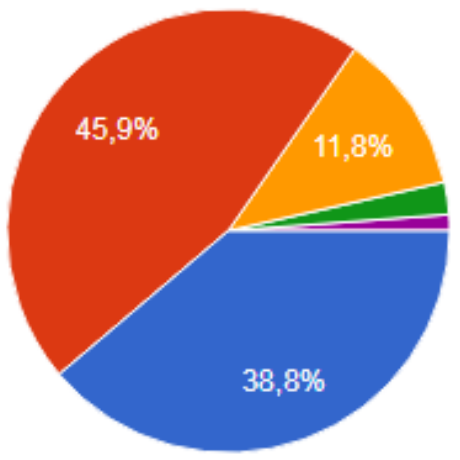

Nunca (0)

Menos de una vez al mes (1)

Mensualmente (2)

Semanalmente (3)

A diario o casi a diario (4)

Figura 2 Autocontrol para dejar de beber

Fuente: Cristhian Villafuerte, 2018.

El 45,9\% de los conductores que beben, al menos una vez al mes no pueden controlar la cantidad de alcohol que ingirieron, en cambio el 38,8\% de los conductores que beben no tienen ese problema y un $11,8 \%$ mensualmente tiene un problema de autocontrol.

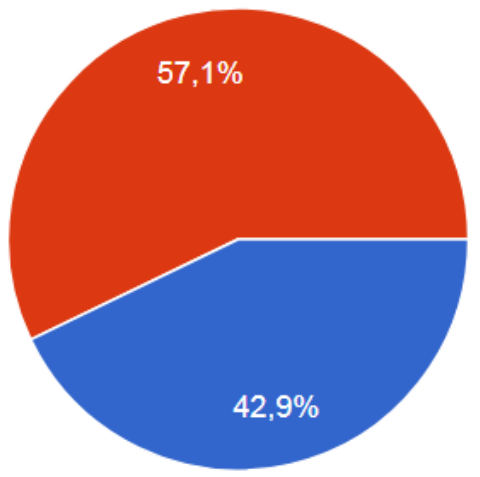

Figura 3 Incomodidad por la forma de beber

Fuente: Cristhian Villafuerte, 2018.

\section{Diseño de sistema electrónico}

Se pretende expandir este proyecto para el transporte interprovincial de esta manera ayudar a reducir los siniestros de tránsito puesto que en este último tiempo las cifras de accidentes se han disparado considerablemente ya que a lo que va del 2018 ya rebasó las cifras del 2017. Con este proyecto se procura minimizar los accidentes registrados por dichas causas que sean por la ingesta de alcohol por parte de los conductores

\section{Políticas de aplicación}

\section{Vehículos más seguros}

Dentro de esta política se maneja una factibilidad legal dentro de un marco normativo a nivel nacional del ente rector del transporte como es la Agencia Nacional de Tránsito para justificar 
de manera legal la implementación de sistema electrónico para el bloqueo de vehículos de transporte público basados en la medida de alcohol por litro de sangre en los conductores en el transporte público en el Cantón Riobamba.

\section{Mecanismo sensorial}

Sensores: El sensor de alcohol MQ-3 analizó el nivel concentrado de alcohol en el aliento en una persona con tres tipos de bebidas:

- $\quad$ Cerveza

- $\quad$ Preparado de caña (puro)

- Vino
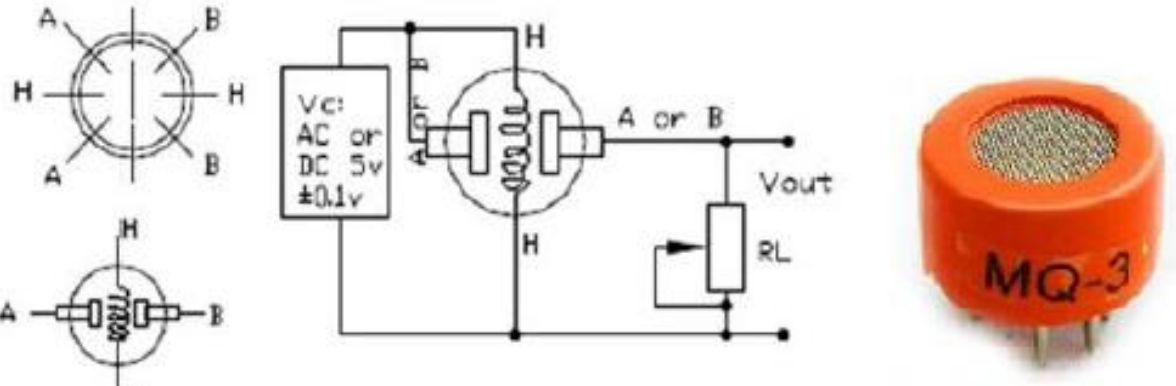

Figura 4 Esquema del Sensor

Fuente: (Hanwei Electronics Co.Ltd, 1998)

\section{Mecanismo de procesamiento}

Plataformas: Para este proyecto se ha elegido una plataforma de hardware libre, buscando las características específicas que se adapten al objetivo y requerimiento del objetivo, en el mercado se pueden encontrar diversas plataformas, pero al comparar las ventajas y desventajas resulta óptimo ocupar "Arduino" por su amplia gama de beneficios. Este es una plataforma de hardware de código abierto que basta con un conocimiento básico de programación en $\mathrm{C}++$ logrando cumplir con la tarea del prototipo.

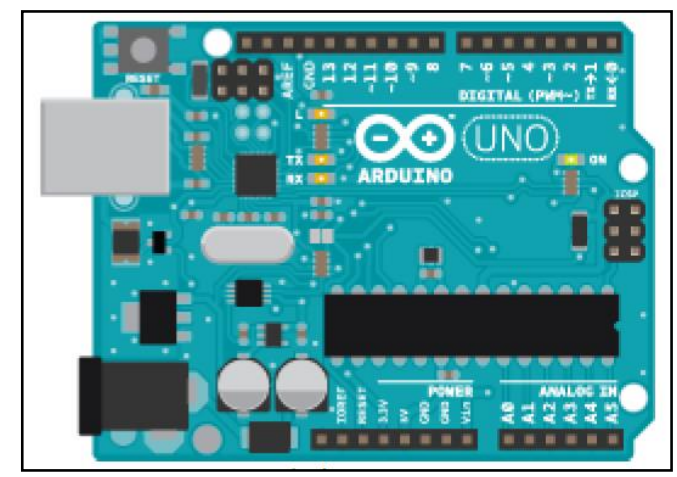

Figura 5 Arduino Uno

Fuente: (Arquino, 2017) 
Interfaz de usuario: La interfaz del usuario es un eje de acción el cual consiste en enviar un mensaje de alerta mediante un SMS al dispositivo móvil asignado con anterioridad, el cual se pretende que sea por parte de la empresa/cooperativa el presidente y por parte de la entidad competente reguladora la ANT o la Dirección de Movilidad Transito Transporte y Seguridad Vial del Gobierno Autónomo Descentralizado Municipal del Cantón Riobamba.

Interfaz del ente regulador: La interfaz que visualizará la ANT o la Dirección de movilidad del GADM Riobamba dependerá del dispositivo donde quieran recibir las notificaciones, para este proyecto se receptará en un dispositivo móvil.

Interfaz usuario conductor: La interfaz que podrá visualizar el conductor del bus será la pantalla Led del dispositivo donde verificará el estado del sistema, como también la permisión o bloqueo del vehículo una vez hecha la prueba.
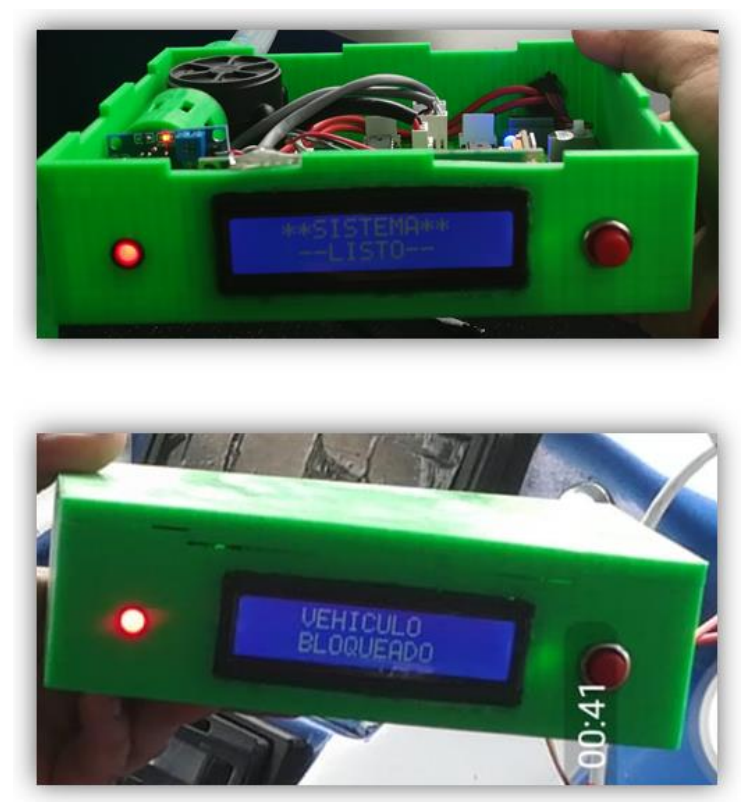

Figura 6 Interfaz conductor

Fuente: Cristhian Villafuerte, 2018

\section{Sistema de comunicación}

La tecnología que se utiliza en este sistema es tecnología 2G que abarca a GSM la cual es simple pero efectiva ya que la tecnología superior tiene más ventajas y servicios, pero el costo y la configuración con su programación no será aprovechada puesto que solo se requiere actividades básicas, especificas, pero muy trascendentales para el control de los conductores, la cual requiere la capacidad de enviar y recibir mensajes de textos.

\section{Cálculo del Chi- cuadrado}

En la siguiente tabla podremos ver las 29 mediciones de miligramos de alcohol por litros de sangre de diversos tipos de bebidas embriagantes mediante el dispositivo electrónico con la participación de seis individuos con diferente peso corporal durante dos días, en las cuales 
probaron soplando de manera directa en el sensor MQ-3 y el segundo día por medio de la manguera y silbato para corroborar si marca los valores reales y si existe alguna alteración al exhalar por medio de la manguera.

Tabla ¡Error! No hay texto con el estilo especificado en el documento.1: Cálculo de Chi Cuadrado

\begin{tabular}{|c|c|c|c|c|}
\hline MUESTRAS & $\begin{array}{l}\text { Tipo de } \\
\text { alcohol }\end{array}$ & $\begin{array}{l}\text { FRECUENCIA } \\
\text { OBSERVADA }\end{array}$ & $\begin{array}{l}\text { FRECUENCIA } \\
\text { ESPERADA }\end{array}$ & $\frac{(F O-F E)^{2}}{F E}$ \\
\hline 1 & Cerveza & 0,14 & 0,13 & 3,8777 \\
\hline 2 & Cerveza & 0,13 & 0,13 & 0,0008 \\
\hline 3 & Cerveza & 1,10 & 0,13 & 7,2377 \\
\hline 4 & Cerveza & 0,14 & 0,13 & 0,0008 \\
\hline 5 & Cerveza & 0,32 & 0,13 & 0,2777 \\
\hline 6 & Cerveza & 0,15 & 0,13 & 0,0031 \\
\hline 7 & Cerveza & 0,15 & 0,13 & 0,0031 \\
\hline 8 & Cerveza & 0,16 & 0,13 & 0,0069 \\
\hline 9 & Cerveza & 0,14 & 0,13 & 0,0008 \\
\hline 10 & Cerveza & 0,24 & 0,13 & 0,0931 \\
\hline 11 & Cerveza & 0,19 & 0,13 & $\mathbf{0 , 0 2 7 7}$ \\
\hline 12 & Cerveza & 0,20 & 0,13 & $\mathbf{0 , 0 3 7 7}$ \\
\hline 13 & Cerveza & 1,00 & 0,13 & 5,8223 \\
\hline 14 & Cerveza & 0,13 & 0,13 & $\mathbf{0 , 0 0 0 0}$ \\
\hline 15 & Cerveza & 0,15 & 0,13 & 0,0031 \\
\hline 16 & Cerveza & 0,25 & 0,13 & 0,1108 \\
\hline 17 & Vino & 0,29 & 0,19 & 0,0526 \\
\hline 18 & Vino & 0,20 & 0,19 & 0,0005 \\
\hline 19 & Vino & 0,19 & 0,19 & 0,0000 \\
\hline 20 & Vino & 0,19 & 0,19 & 0,0000 \\
\hline 21 & Vino & 0,21 & 0,19 & 0,0021 \\
\hline 22 & Vino & 0,21 & 0,19 & 0,0021 \\
\hline 23 & Vino & 0,18 & 0,19 & 0,0005 \\
\hline 24 & Punta & $\mathbf{0 , 5 0}$ & 0,45 & 0,0056 \\
\hline 25 & Punta & $\mathbf{0 , 5 0}$ & 0,45 & 0,0056 \\
\hline 26 & Punta & $\mathbf{0 , 4 0}$ & 0,45 & 0,0056 \\
\hline 27 & Punta & 0,41 & 0,45 & 0,0036 \\
\hline 28 & Punta & 0,43 & 0,45 & 0,0009 \\
\hline 29 & Punta & 0,46 & 0,45 & 0,0002 \\
\hline
\end{tabular}

Elaborado por: Cristhian Villafuerte 2019 


\section{Cálculo de Grados de libertad con un nivel de confianza del $5 \%$}

$\alpha=0,05$ (Nivel de confianza)

Grados de libertad $=\mathrm{k}-1$

Donde k es el número de categorías

Grados de libertad $=29-1=28$

Tabla 2 Distribución Chi cuadrado $\mathrm{X}^{2}$

\begin{tabular}{|r|r|r|r|r|r|r|r|r|r|}
\hline $\mathbf{v} / \mathbf{p}$ & $\mathbf{0 , 0 0 1}$ & $\mathbf{0 , 0 0 2 5}$ & $\mathbf{0 , 0 0 5}$ & $\mathbf{0 , 0 1}$ & $\mathbf{0 , 0 2 5}$ & $\mathbf{0 , 0 5}$ & $\mathbf{0 , 1}$ & $\mathbf{0 , 1 5}$ & $\mathbf{0 , 2}$ \\
\hline $\mathbf{1}$ & 10,827 & 9,1404 & 7,8794 & 6,635 & 5,024 & 3,842 & 2,706 & 2,072 & 1,642 \\
\hline $\mathbf{2}$ & 13,815 & 11,983 & 10,597 & 9,21 & 7,378 & 5,992 & 4,605 & 3,794 & 3,218 \\
\hline $\mathbf{3}$ & 16,266 & 14,32 & 12,838 & 11,34 & 9,348 & 7,815 & 6,251 & 5,317 & 4,642 \\
\hline $\mathbf{4}$ & 18,466 & 16,424 & 14,86 & 13,28 & 11,14 & 9,488 & 7,779 & 6,745 & 5,989 \\
\hline $\mathbf{5}$ & 20,515 & 18,385 & 16,75 & 15,09 & 12,83 & 11,07 & 9,236 & 8,115 & 7,289 \\
\hline $\mathbf{6}$ & 22,458 & 20,249 & 18,548 & 16,81 & 14,45 & 12,59 & 10,64 & 9,446 & 8,558 \\
\hline $\mathbf{7}$ & 24,321 & 22,04 & 20,278 & 18,48 & 16,01 & 14,07 & 12,02 & 10,75 & 9,803 \\
\hline $\mathbf{8}$ & 26,124 & 23,774 & 21,955 & 20,09 & 17,53 & 15,51 & 13,36 & 12,03 & 11,03 \\
\hline $\mathbf{9}$ & 27,877 & 25,463 & 23,589 & 21,67 & 19,02 & 16,92 & 14,68 & 13,29 & 12,24 \\
\hline $\mathbf{2 1}$ & 46,796 & 43,775 & 41,401 & 38,93 & 35,48 & 32,67 & 29,62 & 27,66 & 26,17 \\
\hline $\mathbf{2 2}$ & 48,268 & 45,204 & 42,796 & 40,29 & 36,78 & 33,92 & 30,81 & 28,82 & 27,3 \\
\hline $\mathbf{2 3}$ & 49,728 & 46,623 & 44,181 & 41,64 & 38,08 & 35,17 & 32,01 & 29,98 & 28,43 \\
\hline $\mathbf{2 4}$ & 51,179 & 48,034 & 45,558 & 42,98 & 39,36 & 36,42 & 33,2 & 31,13 & 29,55 \\
\hline $\mathbf{2 5}$ & 52,619 & 49,435 & 46,928 & 44,31 & 40,65 & 37,65 & 34,38 & 32,28 & 30,68 \\
\hline $\mathbf{2 6}$ & 54,051 & 50,829 & 48,29 & 45,64 & 41,92 & 38,89 & 35,56 & 33,43 & 31,79 \\
\hline $\mathbf{2 7}$ & 55,475 & 52,215 & 49,645 & 46,96 & 43,19 & 40,11 & 36,74 & 34,57 & 32,91 \\
\hline $\mathbf{2 8}$ & 56,892 & 53,594 & 50,994 & 48,28 & 44,46 & 41,34 & 37,92 & 35,72 & 34,03 \\
\hline $\mathbf{2 9}$ & 58,301 & 54,966 & 52,336 & 49,59 & 45,72 & 42,56 & 39,09 & 36,85 & 35,14 \\
\hline $\mathbf{3 0}$ & 59,702 & 56,333 & 53,672 & 50,89 & 46,98 & 43,77 & 40,26 & 37,99 & 36,25 \\
\hline $\mathbf{3 1}$ & 61,098 & 57,692 & 55,003 & 52,19 & 48,23 & 44,99 & 41,42 & 39,12 & 37,36 \\
\hline
\end{tabular}

Fuente:https://es.slideshare.net/pilosofando/tabla-chi-cuadrado-16923626

\section{Conclusiones.}

- Las tecnologías aplicadas al transporte cada vez son más importantes al cumplir con funciones como asistentes en la conducción por lo que para un mejor control se ha considerado variables que permitan evitar la manipulación y alteración de los resultados del sistema electrónico como son: trasmisión de datos telefónicos, el sensor de alcohol, relé de acceso o bloqueo de ignición, relé de acceso o bloqueo de la bomba de combustible, un Arduino quien tomará una decisión luego de procesar la información del monitoreo de las condiciones biometrías del conductor y las medidas máximas permisibles como lo dispone el código orgánico penal para que de esta manera precautelar la integridad del mismo y de los demás usuarios viales. 
- El presente sistema electrónico sistema electrónico para el bloqueo de vehículos de transporte público basados en la medida de alcohol por litro de sangre en los conductores cumple con su cometido el cual es monitorear de los medios biométricos del conductor donde se analizan el estado de sobriedad de los conductores para dar un aviso de alerta en caso de marcar un valor en el dispositivo.

- El test realizado por alcoholímetro tiene una precisión con un grado de error mínimo al momento de hacer la medición por lo que es fiable al estar dentro de las normativas de la legislación vigente. Este sistema tiene una tecnología abierta para realizar mejorías, actualizaciones de sistema e incluir nuevos servicios como un módulo biométrico para reconocimiento de huella dactilar, y un módulo de GPS para der un seguimiento a tiempo real de la ubicación de las unidades de buses.

- Al momento de contar con tecnología asistencial aplicada al transporte en un ámbito de control y seguimiento se puede potencializar el programa mediante programas de concienciación de seguridad vial como parte estratégica en programas macros como es el pacto nacional de seguridad vial. Donde se busca potenciar el dispositivo mediante inversión local y agregar funciones que eviten la alteración del sistema de control

\section{Referencias Bibliográficas.}

- $\quad$ N. (2017). Siniestros por causas probables a nivel nacional.

- Agencia Nacional de Tránsito. (23 de octubre de 2013). Plan Nacional de Seguridad Vial.

- Arquino. (2017). Arduino. Recuperado el 2018 de octubre de 31, de https://www.arduino.cc/

- Asamblea Constituyente. (07 de agosto de 2008). Ley orgánica de transporte terrestre tránsito y seguridad vial. (R. O. 398, Ed.) Recuperado el 12 de noviembre de 2018

- Censos, I. N. (22 de julio de 2013). Consumo de alcohol en el ecuador. Ecuador.

- Espinosa Arcentales, M., Salazar, C., \& Asanza, V. (enero de 2016). Diseño de un alcoholimetro digital acoplado a un LDR que permite analizar los parámetros de frecuencia cardiaca utilizando un Arduino mega. Guayaquil, Ecuador.

- Guache Garcell, H., Martinez Quesada, C., \& Gutiérrez García, F. (2007). Efecto del alcohol en la capacidad de conducción de vehículos automotores. Revista Cubana Salud Pública, 6.

- Hanwei Electronics Co.Ltd. (1998). MQ-3 Sensor Gas. Recuperado el 2018 de noviembre de 09 , de http://www.hwsensor.com.

- Lara Tapia, J. C. (marzo de 2006). Conceptos básicos de telefonía celular. Recuperado el 19 de septiembre de 2018, de https://repository.uaeh.edu.mx/bitstream/bitstream/handle/123456789/10895/Conce ptos $\% 20$ telefonia\%20celular.pdf?sequence $=1$.

- Martin, A. (31 de 08 de 2016). ¿Cómo funciona un alcoholímetro? 
- Martínez Pons, J., \& de Prada Pérez de Azpeitia, F. (enero - marzo de 2003). Alcohol y alcoholímetros Historia, fundamentos científicos y aplicación didáctica. Dialnet, 53-62.

- Ministerio de Interior. (2014). El alcohol y la conducción. Dirección General de Tráfico. Madrid, España. Obtenido de www.dgt.es

- Ministerio de Justicia, D. H. (2014). Código Orgánico Integral Penal. Quito, Ecuador.

- $\quad$ Muñoz Vera, K. A. (2011). Análisis de la Tecnología Long Term Evolution (LTE) para su posible implementación en el Ecuador. Sangolqui, Pichincha, Ecuador. Recuperado el 21 de septiembre de 2018, de http://repositorio.espe.edu.ec/handle/21000/4700.

- $\quad$ Normalización, I. E. (2011). Reglamento Técnico Ecuatoriano. Quito.

- Pastrano Badillo, L. G. (2017). Diseño e implementación de un sistema electrónico para conducción segura, basado en monitorización de señales biométricas, utilizando tecnologías GSM, GPRS. Riobamba, ecuador.

- Rappaport, T. (2011). Wireless Communications Principles and Practice. Prentice Hall, 14-17.

- Rodríguez Parrón, M. (2006). Aproximación conceptual e identificación de predictores de riesgo teóricos en jóvenes conductores: un punto de partida para contribuir en la reducción de accidentes de tráfico. 189-203. Barcelona, Spain. Recuperado el 28 de septiembre de 2018

- Shanghai SIM COM Wireless Solutions Ltd. (20 de septiembre de 2013). SIM800L_Hardware_Desing_V1.00.

- Vera Calva, M. A., \& Corona Balderas, L. E. (octubre de 2015). Diseño e implementación de un sistema con alcoholímetro para impedir la marcha de un automóvil cuando el conductor rebase el nivel permitido.

- $\quad$ Andres, V. H. (abril de 2019). Diseño de un sistema electrónico para el bloqueo de vehículos de transporte público basados en la medida de alcohol por litro de sangre en los conductores. Riobamba, Chimborazo, Ecuador 


\section{PARA CITAR EL ARTÍCULO INDEXADO.}

Villafuerte Haro, C., Villacís Torres, S., \& Villafuerte Haro, V. (2019). Diseño de un sistema electrónico para el bloqueo de vehículos de transporte público basados en la medida de alcohol por litro de

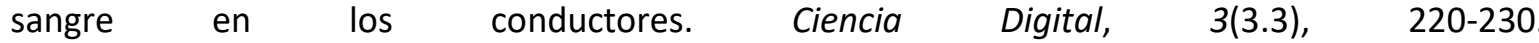
https://doi.org/10.33262/cienciadigital.v3i3.3.801

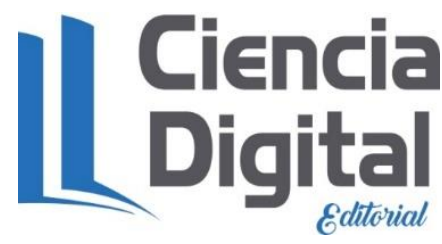

El artículo que se publica es de exclusiva responsabilidad de los autores y no necesariamente reflejan el pensamiento de la Revista Ciencia Digital.

El artículo queda en propiedad de la revista y, por tanto, su publicación parcial y/o total en otro medio tiene que ser autorizado por el director de la Revista Ciencia Digital.
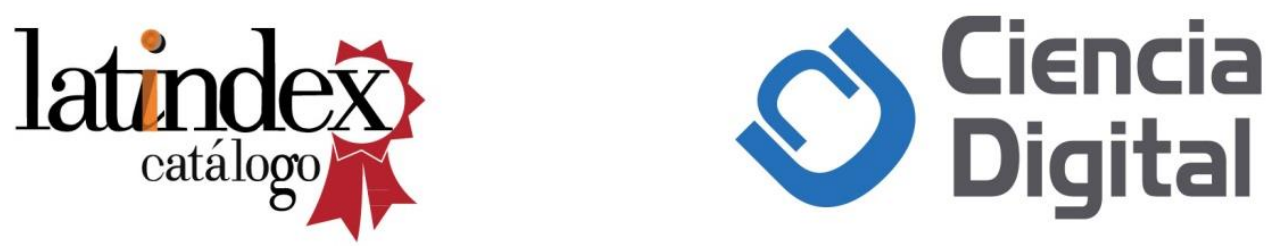